\title{
Degloving injury of the distal aspect of the limb in a horse and preventing bone sequestrum formation using multiple, partial depth cortical fenestration
}

\author{
Filip Kol’vek, Zdeněk Žert
}

University of Veterinary Medicine and Pharmacy in Košice, Equine Clinic, Košice, Slovak Republic

Received July 28, 2020

Accepted February 24, 2021

\begin{abstract}
Exposed or denuded bone is a common complication of wounds of the distal aspect of the limb. This clinical report describes the treatment of an extensive laceration of the right hindlimb of a 12-year-old Hungarian Warmblood Horse gelding, which was managed initially by application of hydrogel dressings to enhance autolytic debridement, subsequently, by cortical fenestration of the denuded cortical bone and by stimulating the production of granulation tissue to cover the exposed bone. An early radiographic sign of sequestrum formation (discrete changes in the outer cortex) was not seen after the injury. Six weeks later, the wound was completely covered with vascularized, healthy-appearing bed of granulation tissue, which was prepared for grafting using the punch technique. A reinforced bandage was applied for the following 7 weeks; the grafts were accepted by granulation tissue. The horse was discharged 20 weeks after admission and the owners were advised to maintain stall rest with hand walking for two weeks. Four years later the owner reported that the wound had completely healed with a good cosmetic outcome and the horse was being used in a carriage.
\end{abstract}

Equine, wound, penetration, metatarsus, punch grafts

Wire, fences, sheet, metal, or other sharp objects in the horse's environment, an entrapment between two immovable objects or during transport usually cause wounds to the distal limbs (Hanson 2004). These degloving injuries of the distal portion of the limb are often associated with extensive soft tissue loss, vascular compromise, crush injury, and severe contamination, making second-intention healing or skin grafting the only options likely to enable successful wound management (Hanson and Schumacher 2016).

Horse's distal limbs are extremely susceptible to damage of the periosteum and underlying bone because of the lack of soft tissue protection. Because the periosteum provides blood supply to the outer one third of the cortical bone, disruption of the periosteum leads to ischaemia of the cortical bone with eventual bone death secondary to these alterations in blood flow. This locally ischaemic and necrotic bone tissue is very susceptible to bacterial colonization and proliferation originating from the initial trauma. Blunt trauma without skin penetration may result in sequestrum formation indicating that bacterial inoculation may be haematogenous. This type of injury is common after a trauma to the cannon bones (Hendrix and Baxter 2005).

The body may be able to resorb the sequestrum or expel it through a draining tract depending on the size of the devitalized bone fragment. Larger fragments usually persist and lead to more extensive clinical disease. The sequestrum can become a chronic nidus delaying the healing process. Because of the lack of vascularization, the immune response at the site is inadequate, leading to a persisting chronic infection (Clem et al. 1988). The affected site most commonly involves some degree of soft tissue swelling and usually has a coexisting draining fistulous tract. There is usually not a significant lameness, but some deep palpation over the sequestered bone generally produces a painful response (Hanson 2008).

Address for correspondence:

Filip Kol'vek

Equine Clinic

University of Veterinary Medicine and Pharmacy in Košice

Komenského 73, 04181 Košice, Slovak Republic 
Desiccation of the superficial layers of exposed bone can lead to sequestrum formation which is one of the most frequent complications of wound healing of limbs in horses. Rapid coverage of the exposed bone with granulation tissue can decrease the healing time and prevent desiccation of the exposed bone. Exposed bone in wounds of the distal limb of horses can result in extensive periosteal new bone growth that can lead to increased wound size and an enlarged circumference of the limb. Cortical fenestration of the exposed bone has been reported in horses, humans and dogs as a means of promoting granulation tissue formation to enhance second-intention healing or provide a vascular bed for skin grafting procedures (Hanson 2004).

The aim of managing a degloving wound on the distal aspect of the limb is to provide the most efficient, cost-effective treatment which may entail the application of a skin graft (Schumacher 2019). A skin graft can be applied to a fresh wound with a blood supply sufficient to support the graft or to a granulating wound, provided that the granulation tissue is healthy and not heavily populated with bacteria. Skin grafts applied to the exposed bone do not survive because the graft cannot be vascularized from the exposed bone. Therefore, the wound can be grafted successfully only after the exposed bone is covered with granulation tissue. The granulation tissue bed to be grafted must be red to pink, smooth, and free of defects (Hanson and Schumacher 2016).

This report describes a successful treatment of a 12-year-old gelding with a laceration of the right metatarsus and cortical fenestration of the exposed bone as a prevention of sequestrum formation.

\section{Case description}

A 12-year-old Hungarian Warmblood Horse gelding injured during the collision with a fence on pasture was presented at the Equine Clinic because of a laceration of the right hindlimb, of an 8-h duration. At admission, the gelding was alert with the wound covered by temporary bandage and abbility to weightbear the limb. Clinical examination revealed a $7 \times 18 \mathrm{~cm}$ degloving injury with exposure of the dorsomedial surface of the third metatarsal bone (Plate I, Fig. 1). Results of blood analyses were within normal values. The horse was sedated with detomidine $(0.01 \mathrm{mg} / \mathrm{kg}$ [IV], Domosedan, OrionPharma) and butorphanol $(0.1 \mathrm{mg} / \mathrm{kg}$ [IV], Butomidor, Richterpharma) and the wound was aseptically prepared for examination. The wound was irrigated with sterile isotonic saline with a small amount of antiseptic ( $0.1-0.2 \%$ povidone-iodine) and explored digitally to establish the extent of injury. There was a visible rupture of the long digital extensor tendon, but the horse was able to compensate for the gait deficiencies. Bone fragments or foreign bodies were not observed. Radiography of the right hindlimb (anterioposterior, lateromedial and oblique views) revealed soft tissue swelling and there was no evidence of any fissures, fracture lines or other bone abnormalities. Regional limb perfusion (RLP) was performed using two grams of amikacin (Selemycin, Medochemie Ltd.) diluted in $60 \mathrm{ml}$ of isotonic saline solution delivered through a catheter inserted into the saphenous vein. The exposed tissue and bone were covered with sterile gauze soaked in hypertonic saline. The intravenous limb perfusion with amikacin was repeated for three following days and the bandage changed daily for 7 days, after which the bandage was changed every other day for 30 days and then less frequently as the wound was covered with granulation tissue. Oxytetracycline was systemically administered (10 mg/kg [IV], Engemycin 10\%, MSD Animal Health) for 3 weeks and nonsteroidal anti-inflammatory drugs (flunixin meglumine, $1.1 \mathrm{mg} / \mathrm{kg}$ [IV], once daily, Flunixin, Norbrook) for 2 weeks. On the third day of hospitalization we proceeded to cortical fenestration of the dorsomedial surface of the metatarsus with a $3.5-\mathrm{mm}$ drill bit penetrating into a depth of 2-3 $\mathrm{mm}$ of the cortical bone (Plate I, Figs 2 and 3). The procedure was performed with the horse standing; chemical restraint was provided 
by a continual rate infusion (CRI) with detomidine and butorphanol. Granulation tissue completely covered the exposed bone 22 days after cortical fenestration (Plate I, Fig. 4). Control X-ray did not reveal sequestrum formation 21 days after the injury (Plate II, Fig. 5). The exuberant granulation tissue was excised as close to the adjacent skin level as possible in combination with topical application of a corticosteroid to stop its formation. Skin grafting technique was performed in order to shorten the healing time of the wound. Pre-operative wound granulation tissue bed preparation was an important factor in the success of the punch grafting method. The goal was to achieve a soft, pink, uniform level of granulation tissue as the recipient site. Topical administration of an antimicrobial drug (penicillin, Penicilin G 5.0; Biotika) was done with gauze soaked in sterile saline for 3 days and bandaging every $12 \mathrm{~h}$. During bandaging, the wound was lavaged with sterile saline and povidone iodide. Graft application was performed with the horse standing. Grafts were harvested from the right side of the neck about $1 \mathrm{~cm}$ apart and in a symmetrical pattern, in an effort to improve the cosmetic appearance of the donor site. Prior to implanting the grafts, subcutaneous tissue was sharply excised from each graft to expose dermal vasculature and encourage plasmatic imbibition and inosculation.

The recipient holes in the wound were created before the grafts were harvested, in a symmetrical pattern, about $6 \mathrm{~mm}$ apart, corresponding to the thickness of the punch grafts. Creation of the 19 holes began distally and proceeded proximally. Cotton-tipped applicator was inserted into the holes to prevent blood clot formation (Plate II, Fig. 6). Grafts were harvested with a $6 \mathrm{~mm}$ diameter biopsy punch needle; the holes were created with a $4 \mathrm{~mm}$ diameter biopsy punch needle. Each graft was inserted into a recipient hole, using tissue forceps and respecting the graft orientation according to the direction of its hair growth. The grafted wound was covered with a non-adherent dressing and then bandaged. During the initial 15 days the grafts became pale with a pink rim of migrating epithelium surrounding them (Plate II, Fig. 7). The wound continued to epithelialize and about $70-75 \%$ of the grafts survived. The gelding was discharged 20 weeks after referral with a partially healed wound (Plate II, Fig. 8) and the owner was advised to maintain stall rest for 2 weeks with a gradually increasing exercise. Two months later the owner reported complete wound healing.

\section{Discussion}

Degloving injuries of the distal portion of the limb are a type of avulsion in which an extensive section of skin is torn from the underlying tissue, severing blood supply to all or a large portion of the skin. The regions most susceptible to degloving injury are the metacarpus and metatarsus because they contain a small amount of soft tissue and are exposed to trauma more often than other regions of the horse's body (Hanson and Schumacher 2016).

Exposed cortical bone, denuded of the periosteum, is subject to desiccation of the superficial layers of the cortex and may result in infectious superficial osteitis and sequestrum formation. The presence of denuded bone in wounds can delay wound healing by prolonging its inflammatory and repair phases. Exposed bone within a wound can delay wound healing directly if the bone becomes infectious vs. septic, or indirectly because its rigid structure can delay the formation of granulation tissue and wound contraction. Injuries involving bones in horses stimulate more periosteal new bone growth than similar wounds in other species or ponies. Periosteal insults from blunt trauma, tendon/joint capsule strain, surgical manipulation, or laceration/degloving injuries may result in extensive periosteal exostosis (Hanson 2004). More extensive periosteal reaction in young compared to adult horses has been attributed to a more active osteoblastic activity of the periosteum in young horses (Caron et al. 1987). The extensive periosteal new bone growth seen in adult horses 
is poorly understood. Discoloration of the exposed bone is not a reliable indicator of sequestrum formation as not all discolored bone develops sequestra (Clem et al. 1988).

Radiographic signs of osteitis are not detectable until at least 7-14 days after the injury when subtle lucent areas may be seen in the cortex. These may be restricted to lucent lines parallel to the margin of the bone, in the outer one third of the cortex, followed by the development of periosteal reaction and more diffuse areas of reduced opacity in the cortex. Earlier diagnosis of infectious osteitis may be possible using ultrasonography. In some cases, this initial appearance progresses to sequestrum formation; a central opaque piece of bone (the sequestrum) is surrounded by a lucent zone (purulent material or granulation tissue) which in turn is bordered by more radiopaque bone (the involucrum). Periostitis may develop proximal and distal to the sequestrum, but does not involve the sequestrum itself. Although antimicrobial therapy may control clinical signs, removal of the sequestrum is usually required for complete recovery (Butler et al. 2017).

Despite the common occurrence of exposed bone associated with trauma to the distal aspect of the limb, there has been little investigation into methods of stimulating coverage of the granulation tissue over the exposed bone in horses. Granulation tissue plays a very important role in second-intention healing because it provides a barrier to infection and mechanical trauma for the underlying tissues. Healthy granulation tissue is resistant to infection and provides a moist surface for epithelialization. The delay in wound healing caused by exposed bone has incited the search for different methods to promote granulation tissue coverage of bone in other species (Han s on 2004). In humans, head trauma, thermal injury, and surgical oncology often results in exposed bone of the cranium (Latenser et al. 1991; Bradley et al. 1998). In these cases, the outer cortex of the exposed portion of the cranium is fenestrated with drill holes, burrs, or laser to expose the medullary cavity from which granulation tissue grows to cover the exposed bone (Cabbage et al. 1982; Bailin and Wheeland 1985; Latenser et al. 1991). Likewise, exposed cortices of long bones in humans have been fenestrated with drill holes to promote granulation tissue formation (Brown 1979). It has been suggested that the drill holes promote healing by allowing osteogenic factors from the medullary cavity access to the wound, or they may enhance healing of the bone and soft tissue by a non-specific response known as "the regional acceleratory phenomenon" (Specht and Colahan 1990). Cortical fenestration combined with drugs that promote topical granulation tissue may accelerate granulation tissue coverage compared to control wounds, however, further investigation is needed. Drilling holes in the cortex of the second metacarpal bone in experimentally created wounds in dogs resulted in clot formation over the bone that promoted granulation tissue formation and may have protected the bone's outer layers from desiccation (Lee et al. 1987). The effects of cortical fenestration were evaluated in experimentally created wounds of the distal aspect of the limb of horses (Johnson 2000). Cortical fenestrated wounds became covered with granulation tissue earlier than control wounds and fenestration had no significant effect on sequestrum formation. The granulation tissue growing directly from the bone surface also contributed to granulation tissue formation. When the wounds are not large $(<6 \times 6 \mathrm{~cm})$, it may be difficult to realize a significant contribution from the granulation tissue growing from the cortical fenestration sites alone. Cortical fenestration may also be beneficial if it is used with other methods of promoting granulation tissue (Johns on 2000). Superficial layers of the cortical bone could be rasped with a bone rasp to achieve bleeding surface (Hans on and Schumacher 2016) and formation of granulation tissue on the surface of the exposed bone cortex could be stimulated by applying calcium alginate (Hendrickson 2015).

Degloving wounds of the dorsal surface of the metacarpus/metatarsus are frequently accompanied by laceration of the associated extensor tendon(s). Although the management of an extensor tendon transection can be challenging in the short term, these wounds 
typically heal by the formation of one large mass of scar tissue that incorporates the tendon and surrounding soft tissues. A lacerated extensor tendon generally does not require tenorrhaphy, unless the laceration is in the region of the fetlock, because a tendon lacerated at that site does not heal readily unless its ends are apposed. A recent report suggests that horses with combined laceration of the lateral and long digital extensors in the hindlimb may benefit from suturing the tendon to the periosteum on the dorsal surface of the third metatarsal bone. No clear association has been identified between the prognosis for a horse with a laceration of a digital extensor tendon and factors such as duration of injury, tenorrhaphy, cast application, breach of a synovial structure, and whether the laceration to the tendon was partial or complete (Dahlgren 2016). In our case, the wound was old, the ends of the long digital extensor tendon were contracted, and no tenorrhaphy was possible. In the case of fresh wounds, reconstruction of the tendons can help to prevent the formation of a sequestrum.

Regardless of the method used for the closure of wounds over exposed bone, formation of healthy granulation tissue in the wound is required. Wound healing over exposed bone relies on the same cellular and humoral interactions present in the healing of other superficial wounds. The inflammatory, debridement, and repair phases work in concert to prepare the wound bed for granulation tissue formation, contraction, epithelialization, and maturation but the presence of exposed bone in wounds may directly or indirectly delay healing. Special attention must be paid to identifying or ruling out the presence of a bone sequestrum when a degloving injury to the distal limb has exposed underlying bone because healing cannot proceed normally until the sequestrum is resolved.

\section{References}

Bailin PL, Wheeland RG 1985: Carbon dioxide (CO2) laser perforation of exposed cranial bone to stimulate granulation tissue. Plast Reconstr Surg 75: 898-902

Bradley DM, Swaim SF, Stuart SW 1998: An animal model for research on wound healing over exposed bone. Vet Com Orthop Traumatol 11: 131-135

Brown PW 1979: The fate of exposed bone. Am J Surg 137: 464-469

Butler JA, Colles CM, Dyson SJ, Kold SE, Poulos PW 2017: The metacarpal and metatarsal regions. In: (Ed. 4): Clinical Radiology of the Horse. John Wiley \& Sons, 239 p.

Cabbage EB, Korock SW, Malik PA 1982: Skin grafting denuded skull. Ann Plas Surg 8: 318-321

Caron JP, Barber SM, Doige CE, Pharr JW 1987: The radiographic and histologic appearance of controlled surgical manipulation of the equine periosteum. Vet Surg 16: 13-20

Clem MF, Debowes RM, Yovich JV, Douglasset JP, Bennett SM 1988: Osseous sequestration in horses, a review of 68 cases. Vet Surg 17: 2-5

Dahlgren LA 2016: Tendon and Paratenon Lacerations. In: Theoret C, Schumacher J (Eds): Equine wound management. Third edn. John Wiley \& Sons, 560 p.

Hanson RR 2004: Management of avulsion wounds with exposed bone. Clin Tech Equine Pract 3: 188-203

Hanson RR 2008: Complications of equine wound management and dermatologic surgery. Vet Clin North Am Equine Pract 24: 663-696

Hanson RR, Schumacher J 2016: Degloving injuries of the distal aspect of the limb. In: Theoret C, Schumacher J (Eds): Equine wound management. Third edn. John Wiley \& Sons, $560 \mathrm{p}$.

Hendrickson DA 2015: What you should and should not put in or on the wound. In: Proceedings of the $61^{\text {st }}$ Annual Convention of the American Association of Equine Practitioners, pp. 9-12

Hendrix SM, Baxter GM 2005: Management of complicated wounds. Vet Clin North Am Equine Pract 21: 217-230

Johnson RJ 2000: The effects of cortical fenestration on second intention healing of wounds over exposed bone of the distal aspect of the limb of horses. Master's Thesis, Auburn University

Latenser J, Snow SN, Mohs FE, Weltman R, Hruza G 1991: Power drills to fenestrate exposed bone to stimulate wound healing. J Dermatol Surg Oncol 17: 265-270

Lee AH, Swaim SF, Newton JC, Brawner WR, McGuire JA 1987: Wound healing over denuded bone. J Am Anim Hosp Assoc 23: 75-84

Schumacher J 2019: Skin grafting. In: Auer JA, Stick JA, Kümmerle JM, Prange T (Eds): Equine Surgery. Fifth edn. Saunders Elsevier, St. Louis, 1896 p.

Specht TE, Colahan PT 1990: Osteostixis for incomplete cortical fracture of the third metacarpal bone: Results in 11 horses. Vet Surg 19: 34-40 
Plate I

Kol'vek F. et al.: Degloving ... pp. 57-61

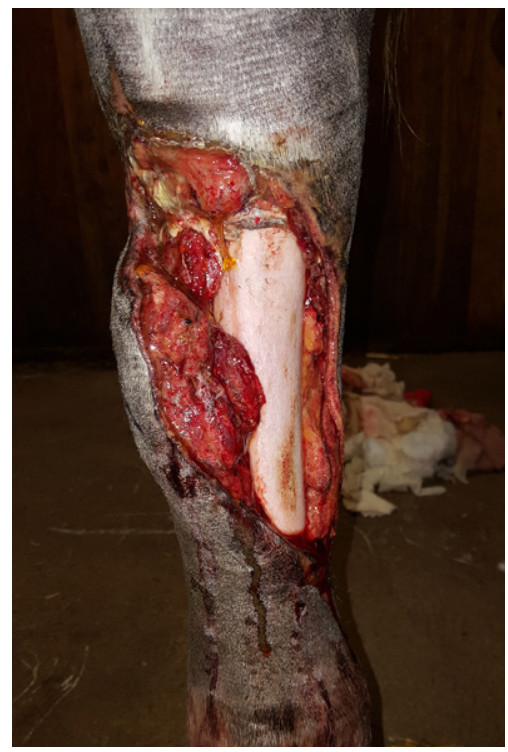

Fig. 1. An extensive degloving wound that exposed the underlying metatarsus in a 12-year-old Hungarian Warmblood gelding. The periosteum was destroyed on a surface of $13 \times 7 \mathrm{~cm}$.

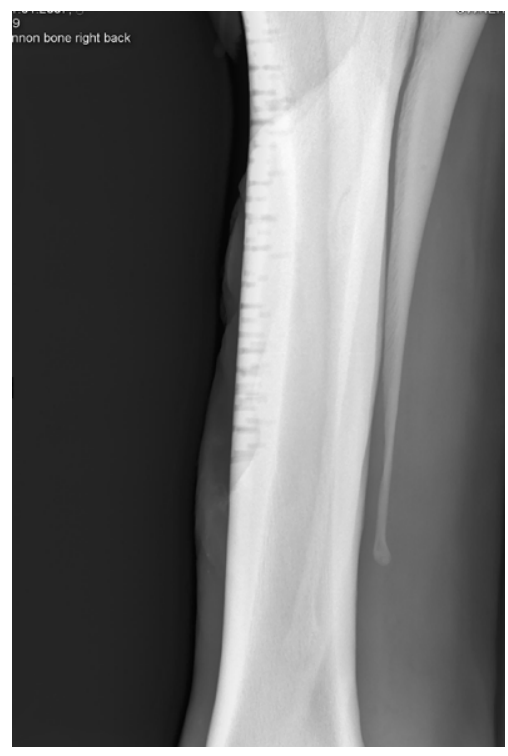

Fig. 3. Lateromedial radiographic view showing several cortical partial thickness holes on the dorsal aspect of the metatarsus after cortical fenestration.

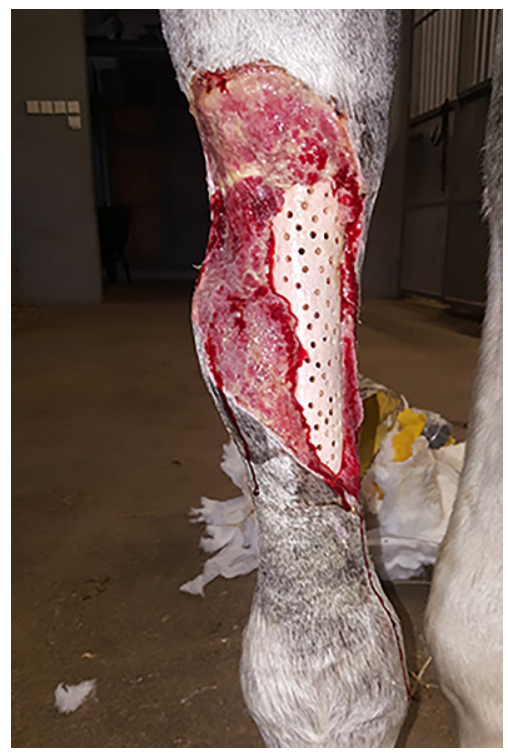

Fig. 2. Cortical fenestration into a depth of 2-3 mm (bleeding holes) on the dorsomedial surface of the metatarsus using a $3.5 \mathrm{~mm}$ drill bit. The cortical fenestration allows granulation tissue to form rapidly over the associated exposed bone and avoid sequestration of the superficial layer of the cortical bone.

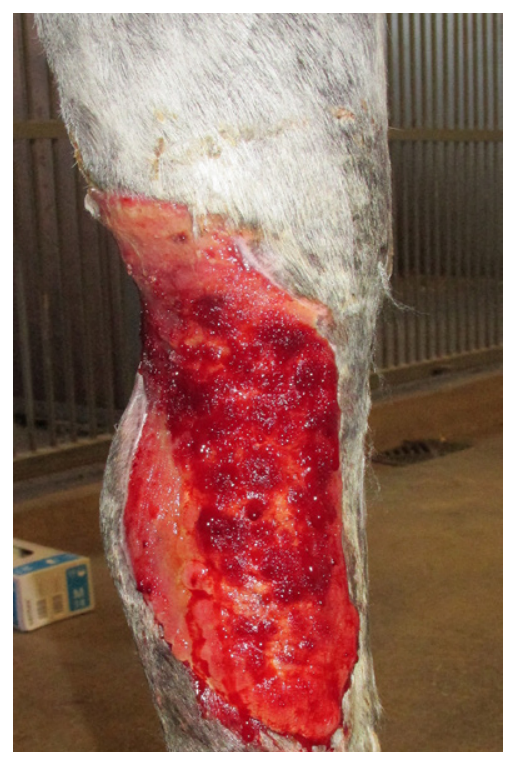

Fig. 4. Granulation tissue has formed at the sites of cortical fenestration in a 22-day-old degloving injury to the dorsal surface of the metatarsus. 
Plate II

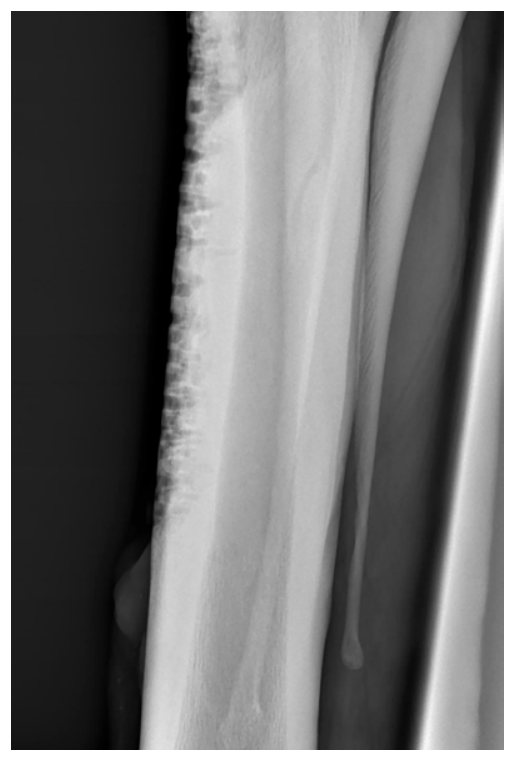

Fig. 5. Dorsolateral-plantaromedial oblique radiographic view of the right metatarsus 21 days after injury. The uppermost layer of the cortex is irregular with reduced opacity of the cortical bone on the dorsal aspect of the metatarsus.

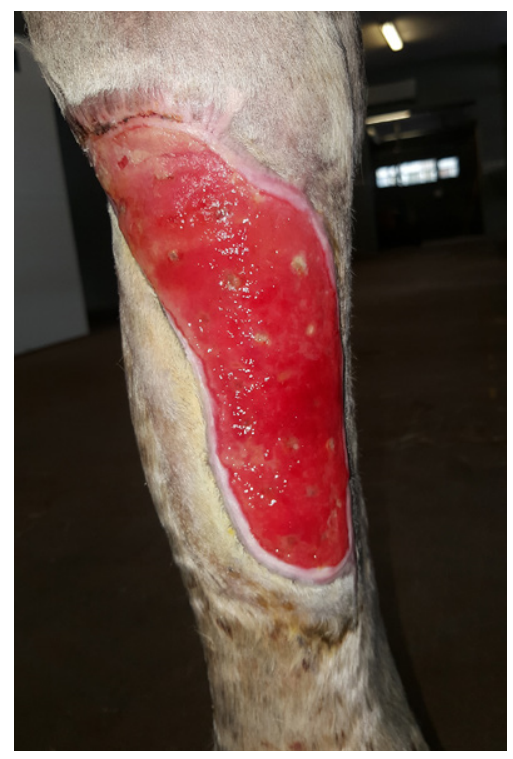

Fig. 7. Fifteen days after the implantation of punch grafts. The punch grafts are evident as pale pink islands within the granulation tissue. Epithelialisation is evident.

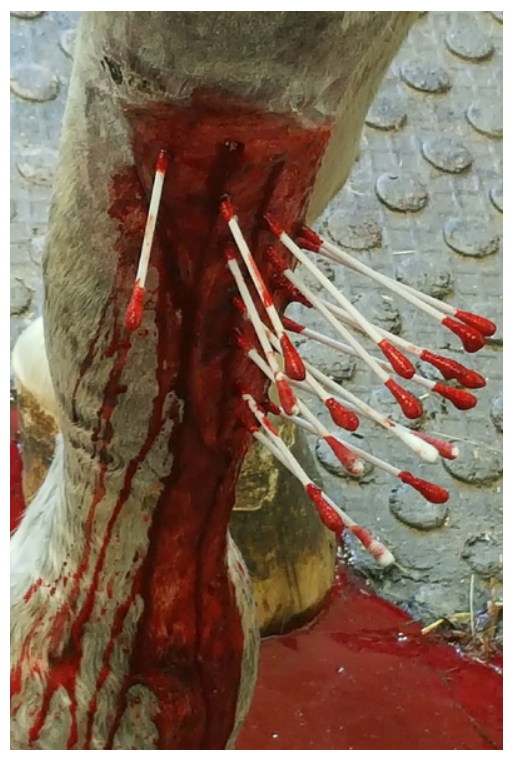

Fig. 6. Recipient graft bed of the dorsomedial metatarsus. Once the $6 \mathrm{~mm}$ biopsy incisions were made in the granulation tissue, cottontipped applicators are placed into each site to control haemorrhage until donor grafts are implanted.

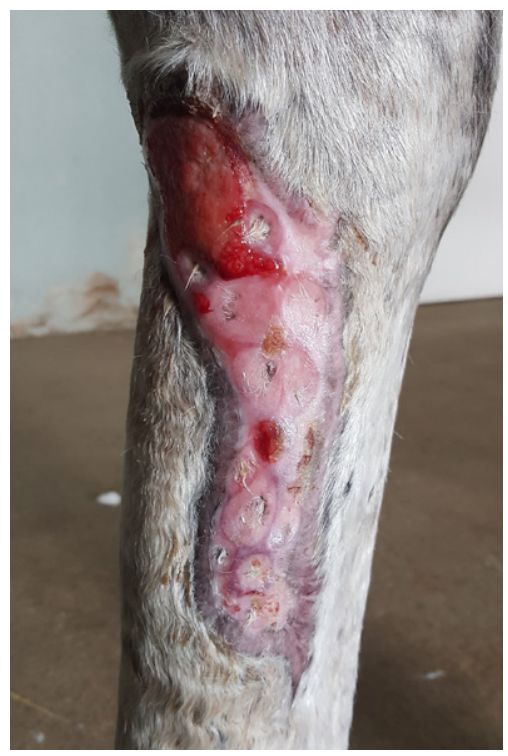

Fig. 8. Partial covering of the wound with epithelium originating from both grafts, as well as from the wound edges, 20 weeks following the original injury and 8 weeks after grafting. 